\title{
O projeto de ensino TourIFRS - Conhecendo o câmpus através de um ambiente virtual online
}

Gabriel Paniz Patzer Instituto Federal de Educação, Ciência e Tecnologia do Rio Grande do Sul Câmpus Sertão

Sertão, Brasil

gabriel.patzer@sertao.ifrs.edu.br
Aline Rezendes Girotto

Instituto Federal de Educação, Ciência e Tecnologia do Rio Grande do Sul -

Câmpus Sertão

Sertão, Brasil

23205@aluno.sertao.ifrs.edu.br
Rafael Rovani Tognon

Instituto Federal de Educação, Ciência e Tecnologia do Rio Grande do Sul -

Câmpus Sertão

Sertão, Brasil

22209@aluno.sertao.ifrs.edu.br
Abstract-TourIFRS is an education project that means to allow access to information about the Instituto Federal de Educação, Ciência e Tecnologia do Rio Grande do Sul (IFRS) - Campus Sertão - in a playful way: using a form of game that will be made available in the institution's website. The problem, from which the project drew inspiration, is the lack of knowledge people have about the structures and departments on the campus, given its extent and complexity. That said, the objective of the project is to develop an introduction website for the IFRS Sertão for the public in general, but especially for those that want to join the institute. The project was motivated by the necessity, aggravated by the COVID-19 pandemic, of having an easy way to know the environment without needing to book a visit and go physically to the campus. The expected result of the project is to provide a virtual mean for any user to learn about the structures of the campus in electronic devices like computers, notebooks, smartphones, etc., through a game-like platform with images, text and map. Therefore, the tool Twine was chosen for development, since it allows for the creation of a non-linear story accessible via internet browsers. In addition to the website development, photos and video in high resolution of the institutes environment were acquired, which will be used in the game, considering responsiveness and accessibility, and be made available in an organized way for use in other projects. In order to create the descriptions of the structures and departments, the students got in contact with the institute's servers via e-mail. The project is in execution phase, with the main navigation structure done, the photos and map partially complete and the styling and evaluation of the website waiting for the completion of the descriptions and photos.

Resumo - O TourIFRS é um projeto de ensino que busca proporcionar acesso a informações sobre o Instituto Federal de Educação, Ciência e Tecnologia do Rio Grande do Sul (IFRS) - Câmpus Sertão - de uma maneira lúdica: através de uma espécie de jogo, que será disponibilizado no site da instituição. O problema, no qual o projeto se inspirou, é a falta de conhecimento que as pessoas têm das estruturas e setores do câmpus, dada a extensão e complexidade dele. Diante disso, o projeto tem o objetivo de desenvolver um site de apresentação do IFRS Câmpus Sertão para o público em geral, mas especialmente aqueles que desejam ingressar na instituição. $O$ projeto foi motivado pela necessidade, agravada pela pandemia do COVID-19, de ter uma maneira de fácil acesso de conhecer o ambiente, sem precisar marcar horário de visita e ir fisicamente ao campus. $O$ resultado esperado do projeto é fornecer um meio virtual que possibilite qualquer usuário conhecer as estruturas do campus através de equipamentos eletrônicos como computador, notebook, smartphone, etc, por meio de uma espécie de jogo com imagens, texto e mapa. Para tanto, foi escolhida a ferramenta Twine para o desenvolvimento, já que ela permite a criação de uma história não-linear acessível via navegadores web. Além do desenvolvimento do website, foram adquiridas fotos e vídeos em alta qualidade do ambiente da instituição, que serão utilizadas na ferramenta, considerando características de responsividade e acessibilidade, e disponibilizadas de forma organizada para serem utilizadas em outros projetos. Para a criação das descrições dos ambientes, os alunos entraram em contato via e-mail com os servidores da instituição. $O$ projeto está em fase de execução, contando com a estrutura principal de navegação concluída, as fotos e mapas parcialmente completas e a estilização e avaliação do site aguardando a finalização das descrições e fotos.

Palavras-chave - desenvolvimento de website; front-end; navegação virtual interativa.

\section{INTRODUÇÃO}

O câmpus Sertão do Instituto Federal de Educação, Ciência e Tecnologia do Rio Grande do Sul (IFRS) possui atualmente 15 cursos [1], atendendo mais de 1300 alunos matriculados no início do ano de 2020. Estes cursos vão das áreas de Agricultura e Zootecnia a Informática, contando com instalações adequadas para tais, o que inclui salas de aula, laboratórios, setores de produção agropecuária e culturas vegetais. Toda essa estrutura está distribuída em um espaço de 237 hectares [2], fazendo com que muitos dos alunos, e até servidores, do câmpus não conheçam alguns dos setores, sua localização ou mesmo para que são utilizados. Esse desconhecimento foi agravado pela situação de isolamento social causada pela pandemia da COVID-19, motivo pelo qual o calendário letivo da instituição foi suspenso e, desde março, os alunos não frequentam o câmpus.

Outro fator que influenciou na decisão de iniciar o projeto foi a dificuldade em encontrar informações básicas sobre algumas estruturas e setores da instituição, que possui um organograma bastante complexo para alunos que estão começando o ensino médio, por exemplo. A estrutura escolar com que os alunos ingressantes estão familiarizados normalmente envolve uma direção e uma coordenação, passando por mais um ou outro setor de apoio; todas suas necessidades administrativas podem ser resolvidas na secretaria. Quando ingressam no IFRS eles encontram mais de um diretor, dezenas de coordenadores de setores e 
funções específicas [3]. Eles não conseguem resolver suas questões diretamente com um professor ou coordenador, pois cada setor possui seus processos e responsáveis, com os quais os alunos muitas vezes têm autonomia e responsabilidade de contatar diretamente.

O projeto TourIFRS foi planejado visando apresentar a estrutura do câmpus de uma forma acessível para toda a comunidade acadêmica, além de segura se considerarmos a situação da pandemia,. Com ele, busca-se construir uma plataforma online onde os visitantes poderão visualizar o ambiente através de fotos e vídeos, além de acessar descrições simples de cada setor e curso do câmpus com a possibilidade de navegar para as respectivas páginas do site do câmpus.

É importante salientar, também, que esse projeto dá continuidade a uma atividade iniciada com alunos do ensino médio, que deveriam de alguma forma auxiliar na divulgação do câmpus. A forma escolhida para realizar essa divulgação foi no estilo de um jogo visual novel, ou livro interativo, onde o visitante pode escolher o caminho que deseja percorrer e escolher sua própria história ou, nesse caso, qual parte do câmpus deseja conhecer. O uso de jogos e atividades lúdicas é uma forma de incentivar a leitura para os jovens [4], que com o TourIFRS estariam aprendendo mais sobre a instituição. O desenvolvimento da atividade no ensino médio foi através do Ren'Py Visual Novel Engine, uma ferramenta bastante simples de utilizar que permite a criação de uma visual novel, porém é necessário o download e instalação, ao invés de oferecer um ambiente online.

\section{A. Projeto de ensino}

O objetivo do projeto TourIFRS é oferecer uma ferramenta para navegação que permita conhecer o Câmpus Sertão do IFRS, mas sua maior contribuição será na formação dos estudantes que nele estão trabalhando. Além de atividades que contribuem para a formação oferecida pelo curso superior em que estão inscritos, a participação nesse tipo de projeto permite que os alunos pesquisem áreas fora do foco do curso, nesse caso pode-se citar especificamente a fotografia e edição de vídeos.

O TourIFRS segue as normas de um projeto de ensino da instituição [5], contando com financiamento na forma de bolsas para dois estudantes. Dessa forma, um dos principais objetivos do projeto é colocar os alunos em contato com o desenvolvimento real de um sistema: os alunos seguem uma metodologia de desenvolvimento incremental; realizam toda a codificação do sistema; participam do seu planejamento; participam e registram reuniões de andamento do projeto. Todas essas atividades promovem uma participação ativa, que diversos autores afirmam que aumenta o interesse [6] e motivação [7] dos alunos.

Ambos os alunos bolsistas são estudantes do primeiro semestre do curso de Tecnologia em Análise e Desenvolvimento de Sistemas. Muitas das atividades que eles desenvolvem durante o projeto são conteúdos que eles não estudariam em sua etapa atual do curso, além da experiência adquirida em trabalho cooperativo.

\section{DESENVOLVIMENTO}

Os alunos bolsistas realizam o desenvolvimento do sistema, sob orientação de um professor da instituição. Essa orientação ocorre em reuniões em videochamada de aproximadamente 30 minutos, duas vezes por semana, onde os alunos são responsáveis também por fazer uma síntese da reunião e decisões tomadas. Durante esses encontros cada aluno apresenta o andamento das suas tarefas, define os próximos passos e tira dúvidas que surgiram durante suas pesquisas.

\section{A. Website}

Inicialmente, os alunos pesquisaram sobre jogos e ferramentas para desenvolvimento de jogos no estilo visual novel. A intenção era colocá-los em contato com o tipo de interação desejado e fazer com que escolhessem uma ferramenta adequada para o seu desenvolvimento. Nesse processo, eles fizeram testes com Renpy, Novelity, TyranoBuilder, Twine, Brackets e Phaser. Dessas, foi escolhida a Twine [8] como plataforma para desenvolvimento, por ser de código aberto, possuir interface amigável para desenvolvimento e ser possível construir uma história mesmo com pouco conhecimento de programação. Outra grande vantagem dessa ferramenta, que foi a principal influência para sua escolha, é ela gerar o código em HTML, CSS e Javascript, facilitando sua integração em um website já existente ou hospedagem em um novo domínio. Esperase, ao final do projeto, incluir o resultado do projeto no site da instituição, de modo a facilitar seu acesso a qualquer interessado em conhecer um pouco mais sobre o câmpus.

Uma preocupação importante do projeto, no sentido de permitir esse fácil acesso, é a acessibilidade e responsividade do ambiente. Para tanto, os alunos estão realizando testes com bibliotecas como a Bootstrap [9] e tomam cuidados de acessibilidade como: uso de legendas nos vídeos; esquemas de cores colorblind-friendly; uso de descrições nas imagens. Esta etapa está em fase de planejamento e teste iniciais, não estando integrada à linha de desenvolvimento principal. À medida em que o conteúdo do site for finalizado a etapa de estilização iniciará.

Como uma forma de navegação alternativa à de links em texto, também está sendo construído um mapa interativo com a biblioteca Leaflet [10]. Nesse mapa, que segue a estratégia de mapas coropléticos [11], algumas regiões e as estruturas do câmpus estão destacadas, permitindo o usuário fazer a navegação diretamente até um elemento específico do câmpus. Uma amostra do resultado pode ser vista na figura 1 .

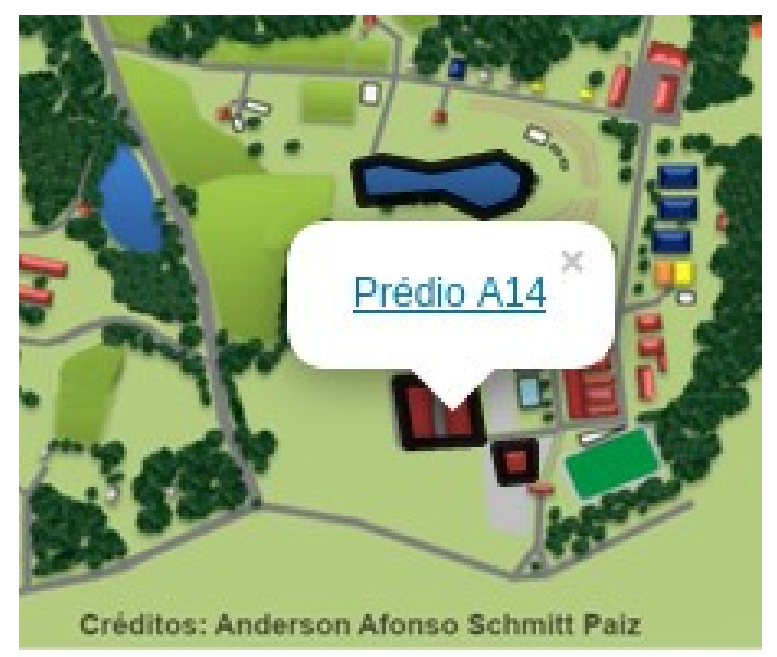

Fig. 1. Destaque do mapa com elementos clicáveis, que apresentam o nome da estrutura e link para navegação. 
Outro importante elemento do desenvolvimento que os alunos têm contato no projeto, mas não nas etapas iniciais do seu curso, é o uso de um sistema de controle de versão. Nesse caso, o desenvolvimento será realizado utilizando a plataforma GitHub [12], incluindo, temporariamente, a hospedagem da ferramenta.

\section{B. Descrições e imagens}

As informações que serão disponibilizadas através do TourIFRS já estão, em parte, disponíveis no site do IFRS Câmpus Sertão. Ainda assim, os alunos buscaram descrições mais completas ou simplificadas com os servidores que atuam em cada setor. Esse levantamento foi realizado através de contato via e-mail, devido às atividades presenciais no câmpus estarem suspensas.

Apesar disso, um dos pontos mais importantes do projeto é o uso de imagens de qualidade para que os usuários possam conhecer e visualizar o ambiente do câmpus. Para tanto, os alunos e orientador visitaram o ambiente para esse levantamento fotográfico, além de solicitar fotos aos servidores via e-mail e no próprio site da instituição.

As fotos adquiridas serão selecionadas, organizadas e pré-processadas. Espera-se que cada setor ou prédio possa contar com duas a quatro fotos cada, de acordo com a variedade de ambientes internos. Ainda por causa da situação de isolamento provocada pela pandemia do COVID-19, não foi possível realizar as fotos de todos os ambientes internos.

A organização das fotos se dará através de um padrão de nomenclatura definido pelos alunos: a largura da foto seguida do código do prédio/estrutura (Fig. 2). Esse padrão busca facilitar a comunicação entre o responsável pelas fotos e o responsável pelo site, para que ambos possam realizar suas atividade concomitantemente, e permitir uma fácil classificação e busca de fotos futuramente, por quem desejar reutilizar esse material.

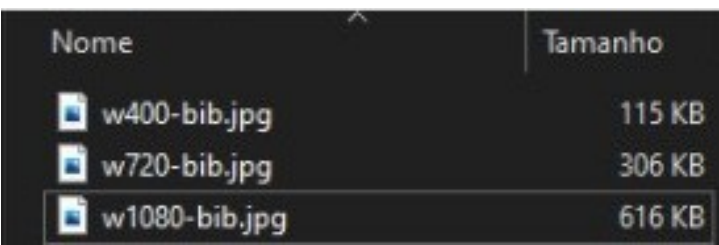

Fig. 2. Amostra da estruturação seguida pelas fotos utilizadas no projeto.

O pré-processamento das fotos envolve um tratamento simples de iluminação, homogeneizando o resultado das fotos que foram adquiridas em diferentes horários do dia, e a criação de fotos em diferentes resoluções, para que seja carregada no navegador do usuário a foto mais adequada à resolução da sua tela. Em qualquer tela ou dispositivo, o usuário poderá clicar na foto para que seja disponibilizada a foto na resolução máxima, para visualização ou download da mesma. A figura 3 apresenta o resultado do tratamento de iluminação realizado.
Juntamente com as fotos, foram realizadas aquisições em vídeo, que serão compostas em um tour pela parte central do câmpus. Para esse vídeo, os alunos elaboraram um roteiro de ordem de gravação e texto a ser apresentado sobre cada prédio. Esse vídeo será o conteúdo em destaque da página inicial do projeto, permitindo que os visitantes conheçam rapidamente as principais áreas do câmpus e, depois, possam utilizar a navegação buscar mais informações ou fotos.

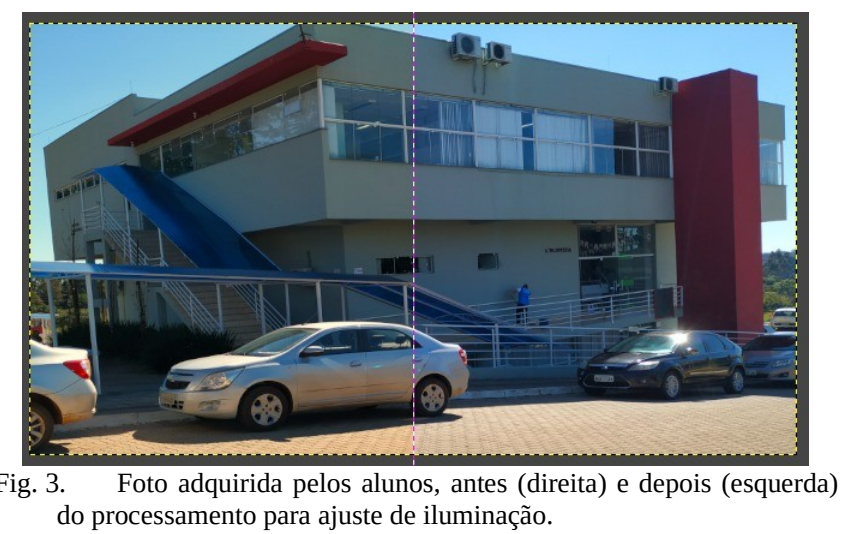

\section{RESUltadOS E DISCUSSÕES}

O projeto, no momento do envio deste artigo, está em fase de execução, com o planejamento principal já definido e tarefas distribuídas, porém com margem para alterações que se julgue necessárias. A maior parte das descrições já foi inserida na ferramenta Twine e algumas fotos como teste de disposição na tela. Um exemplo de tela pode ser visto na figura 4. A estrutura principal de navegação entre os setores, prédios e cursos já está definida, contando com 26 elementos. A estilização das páginas foi realizada de forma básica, sendo necessário ainda um estudo dos esquemas de cores e avaliação por usuários-teste nos diferentes dispositivos que se deseja atender..

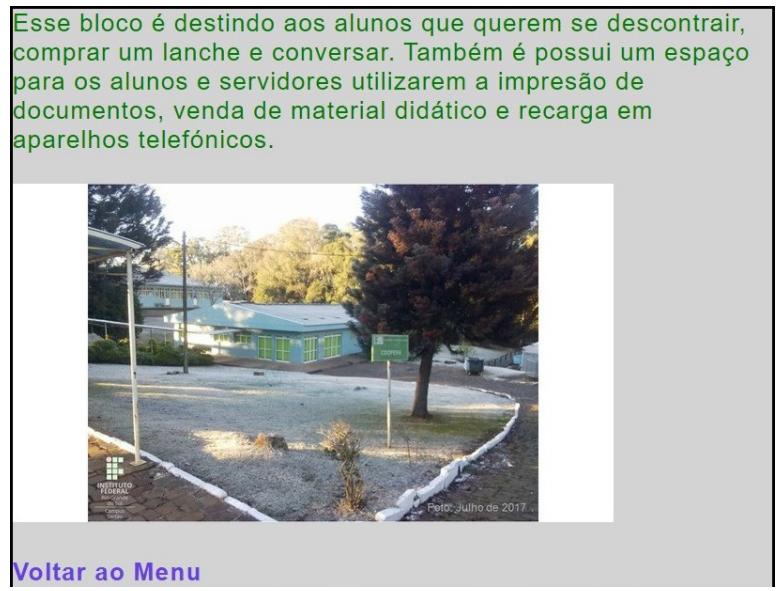

Fig. 4. Tela contendo descrição e imagens da COOPERA, cooperativa de estudantes do Câmpus Sertão.

À estrutura de navegação será adicionado o mapa do campus como forma adicional de navegação. O mapa em si está em processo de construção: aproximadamente 15\% das áreas já foram demarcadas, para que o destaque e ligações sejam realizados.

As fotos foram selecionadas e estão sendo formatadas e padronizadas, com aproximadamente $50 \%$ desse processo concluído. O vídeo tour está com seu material selecionado, 
mas ainda é necessário fazer a seleção de trilha sonora, utilizando fontes livres, a limpeza do áudio dos vídeos originais e a edição montagem do vídeo único.

No momento, as imagens originais estão armazenadas em uma pasta compartilhada na nuvem, mas planeja-se oferecer acesso a elas através de um link direto no site do projeto ou através de uma API. Dessa forma, outras atividades do câmpus podem reutilizar o material já preparado e catalogado neste trabalho.

\section{CONSIDERAÇÕES FINAIS}

As atividades realizadas até este momento no projeto foram muito gratificantes, pois é notável o envolvimento e empolgação dos alunos participantes no projeto. Diversas das tarefas que eles realizaram não seriam feitas no curso superior que estão cursando. Também pode-se citar o exemplo da disciplina voltada para desenvolvimento web, que ocorre no terceiro semestre do curso: os alunos estão tendo uma ótima experiência que irá agregar muito na sua formação muito tempo antes de terem a oportunidade de estudar esses conteúdos no curso.

O acompanhamento em reuniões semanais faz com que os alunos desenvolvam autonomia e desenvoltura para apresentar o trabalho realizado, bem como habilidades de síntese e organização, para que as reuniões não se estendam por um período muito longo de tempo.

Por fim, espera-se que o resultado do projeto traga satisfação e orgulho para os participantes, ao ver uma ferramenta que eles criaram disponível no site de sua instituição e podendo ser utilizada pelo grande público.

\section{AgRADECIMENTOS}

Gostaria de agradecer ao ex-aluno da instituição Anderson Afonso Schmitt Paiz por ceder o mapa desenhado do câmpus e ao IFRS pelo apoio financeiro ao projeto.

\section{REFERÊNCIAS}

[1] IFRS Sertão, “Cursos - Campus Sertão”, Disponível em: https://ifrs.edu.br/sertao/cursos/.

[2] IFRS Sertão, "Histórico - Campus Sertão”,
Disponível em: https://ifrs.edu.br/sertao/institucional/ historico/.

[3] IFRS Sertão, “Organograma - Campus Sertão”, Disponível em: https://ifrs.edu.br/sertao/institucional/ gestao-atual/.

[4] L. M. D. Lopes, K. N. S. Vidotto, E. Pozzebon, L. B. Frigo, "Jogos Digitais Como Recurso De Incentivo À Leitura: Uma Revisão Sistemática”, in Rev. Novas Tecnologias na Educação, Porto Alegre, V. 15, N. 2, Dez. $2017 . \quad$ Disponível em: https://seer.ufrgs.br/renote/article/view/79241/46130.

[5] IFRS Sertão, "Projetos de Ensino - Campus Sertão”, Disponível em: https://ifrs.edu.br/sertao/ensino/projetos-de-ensino-2/

[6] T. Pacievitch, "Evasão Escolar", in InfoEscola., Disponível em: https://www.infoescola.com/educacao/evasaoescolar/.

[7] C. Brandalise, "Uma nova e preocupante evasão escolar”, in ISTOÉ, 20/04/18, Disponível em: https:// istoe.com.br/uma-nova-e-preocupante-evasaoescolar/.

[8] C. Klimas, "Twine / an open-source tool for telling interactive, nonlinear stories”, Disponível em: http://twinery.org/.

[9] Bootstrap, "Bootstrap - the most popular HTML, CSS and JS library in the world.”, Disponível em: https://getbootstrap.com/.

[10] V. Agafonkin, "Leaflet - a Javascript library for interactive maps”, Disponível em: https://leafletjs.com/.

[11] V. Agafonkin, "Interactive Choropleth Map - Leaflet - a Javascript library for interactive maps", Disponível em: https://leafletjs.com/examples/choropleth/.

[12] GitHub Inc., "GitHub: Where the world builds software”, Disponível em: https://github.com/. 